\title{
1 Metal-silicate silicon isotopic fractionation and the composition \\ 2 of the bulk Earth
}

3

4 Frédéric Moynier $^{1}$, Zhengbin Deng ${ }^{1}$, Ariane Lanteri $^{1}$, Rayssa Martins ${ }^{1}$, Marc

5 Chaussidon $^{1}$, Paul Savage ${ }^{2}$, Julien Siebert ${ }^{1,3}$

6 Institut de Physique du Globe de Paris, Université de Paris, CNRS, 1 rue Jussieu, Paris

$7 \quad 75005$, France

$8 \quad{ }^{2}$ School of Earth and Environmental Sciences, University of St Andrews, Irvine

9 Building, St Andrews, KY16 9AL, United Kingdom

$10{ }^{3}$ Institut Universitaire de France, 103 boulevard Saint-Michel, Paris 75005, France

12 moynier@ipgp.fr

14 Abstract:

15 The difference in the $\mathrm{Si}$ isotopic composition between the Earth and primitive 16 meteorites had been used to constrain the amount of Si in the Earth's core. However,

17 there is presently a debate on the magnitude of the isotopic fractionation between metal

18 and silicates as function of temperature based on experimental data. Here, we use a

19 natural sample, an enstatite meteorite, Itqyi, as a natural experiment to determine an

20 independent $\mathrm{Si}$ isotopic fractionation factor between metal and silicate. We determined

21 the temperature of equilibrium between metal and silicate as well as the $\mathrm{Si}$ isotopic

22 composition between the phases. We find that the dependence of $\mathrm{Si}$ isotopes with 
23 temperature to be :

$$
\Delta^{30} S i_{\text {silicate-metal }}=\frac{7.6( \pm 0.7) \times 10^{6}(1 S D)}{T^{2}}
$$

24 Using this dependence of the $\delta^{30} \mathrm{Si}$ with temperature we estimate the bulk Earth $\delta^{30} \mathrm{Si}$ as

25 a function of Si content of the core for different plausible conditions. Even when using

26 the most extreme parameters, we show that the bulk Earth must be isotopically heavier

27 than any chondrites groups. We therefore confirm that core formation alone cannot

28 account for the isotopic difference between the Earth and primitive meteorites. We

29 show that there is no correlation between $\delta^{30} \mathrm{Si}$ and the $\mathrm{Mg} / \mathrm{Si}$ ratio suggesting that

30 forsterite fractionation in the solar nebula may have had only a limited effect, if any.

31 Our new results therefore confirm that volatility should have had a fundamental effect

32 in shaping terrestrial planets chemical composition. 


\section{Introduction}

In order to explain the deficit in density of the Earth's outer core it must contain $\sim 10 \%$ of elements lighter than Fe (Birch, 1964). While, the nature of these lighter elements is still debated, high-pressure high-temperature experiments show that it is most likely composed of O, Si and S (e.g. Badro et al., 2015; Righter and Drake, 2003; Siebert et al., 2013).

Because they are potentially fractionated between metal and silicate, stable isotopes have been employed as independent tracers of core formation and composition (e.g. Bonnand and Halliday, 2018; Creech et al., 2017; Fitoussi et al., 2009; Georg et al., 2007; Hin et al., 2013; Hopp et al., 2018; Mahan et al., 2018; Roskosz et al., 2006; Savage et al., 2015; Shahar et al., 2017; Shahar et al., 2009; Ziegler et al. 2010). In particular it has been demonstrated that the ${ }^{30} \mathrm{Si} /{ }^{28} \mathrm{Si}$ of the silicate Earth is $\sim 0.15 \%$ o (per mil) heavier than those undifferentiated meteorites argued to represent the composition of the bulk Earth (e.g. Armytage et al., 2011; Fitoussi et al., 2009; Georg et al., 2007; Zambardi et al., 2013; Poitrasson 2017). This excess in the heavier isotopes in the silicate Earth is supposedly compensated by an isotopically light core (e.g. Georg et al., 2007) and using estimates of the equilibrium isotopic fractionation between metal and silicate it is possible to calculate the amount of Si that must be stored in the Earth core to explain the observed isotopic difference, assuming no other source of isotopic fractionation (e.g. Hin et al. 2014; Savage et al., 2014; Shahar et al., 2009; Ziegler et al. 2010). However, there is debate over the magnitude of Si isotope fractionation between metal and silicate at equilibrium: the two experimental approaches, based on high-pressure and high-temperature experiments using a piston-cylinder press return 
different results, with Hin et al. (2014) calculating a smaller degree of isotopic fractionation than Shahar et al. (2009) at the temperatures relevant to core formation.

The degree of this difference is significant, as using the Hin et al. (2014) isotopic fractionation factor would either require an unrealistic amount of Si present in the core (whatever chondrites type used as starting composition) to explain the silicate Earth composition, if core formation was the only process which has affected the $\mathrm{Si}$ isotope composition of the BSE since Earth's formation.

An alternative and complementary approach would be to use natural samples that contain equilibrated metal and silicate with measurable amounts of Si partitioned into the metal. However, samples fitting this requirement (metal and silicate that equilibrated at low $\mathrm{fO}_{2}$ conditions) are rare and so far only two samples have been studied (Ziegler et al., 2010). Using two enstatite achondrites, which equilibrated at similar temperatures (1200 K and 1130K), Ziegler et al. (2010) measured the difference in $\mathrm{Si}$ isotopic composition between metal and silicate. Their results are in agreement with the experimental curve determined by Shahar et al. (2009), and fall off that determined by Hin et al. (2014), although, since both meteorites analyzed here, Mount Egerton and Norton County, equilibrated under similar temperature, it only provides one data point to the calibration curve.

The hypothesis behind the above approaches is that the Si isotopic difference between the BSE and chondrites is uniquely controlled by core formation. The fact that an unrealistic amount of Si may need to be stored in the Earth's core (e.g. Fitoussi and Bourdon, 2012; Hin et al., 2014) together with the later discovery of heavy Si isotopic 
composition in angrite meteorites representing the silicate fraction of a small oxidized (with a priori no $\mathrm{Si}$ in its core) de-volatized differentiated parent body suggest that volatility may exert a strong control on the Si isotopic composition of a planet, which have greatly changed our vision on how planet acquired their present chemical composition (e.g. Fitoussi et al., 2009; Hin et al., 2017; Pringle et al., 2014; Sikdar and Rai, 2020; Zambardi et al., 2013; Young et al., 2019). Based on a correlation between $\delta^{30} \mathrm{Si}$ and $\mathrm{Mg} / \mathrm{Si}$, Dauphas et al. (2015) proposed an alternative scenario in which the enrichment in the heavy $\mathrm{Si}$ isotope represent gas-forsterite isotopic fractionation in the solar nebula. The Earth would be relatively depleted in refractory phases (forsterite), decreasing its $\mathrm{Mg} / \mathrm{Si}$ ratio and increasing its $\delta^{30} \mathrm{Si}$. However, this approach needs also an estimate of $\delta^{30} \mathrm{Si}$ of the bulk Earth and therefore relies on the Si isotopic fractionation during core formation.

1 Given the important implications cited above, it is therefore primordial to better constrain the exact $\mathrm{Si}$ metal-silicate isotopic fractionation factor, to establish the maximum effect of core formation on the Si isotopic composition of the Earth's mantle, in order to estimate the composition of the present bulk Earth. Here, we determine the $\mathrm{Si}$ isotopic fractionation between metal and silicate using a recently discovered meteorite, Itqyi. Itqyi is metal-rich and coarse-grained, regarded as an enstatite achondrite (e.g. Patzer et al., 2001; Patzer et al., 2002) which, similar to the samples utilized in Ziegler et al. (2010), equilibrated under very reduced conditions and partitioned several percent of Si into its metal phase. Since there have been very limited studies on Itqyi, we first demonstrate that metal and silicate phases are at equilibrium, 
101 and determine the $\mathrm{fO}_{2}$ and temperature of equilibrium based on elemental partitioning experiments. Finally, we present the Si isotopic composition of the metal and silicate

103 phases. We will show that the temperature of metal/silicate equilibrium is different

104 from the two meteorites used in Ziegler et al. (2010), which will allow us to provide a 105 new independent isotopic fractionation factor at a different temperature, to test the 106 previous estimates of the meta/silicate isotopic fractionation, estimate the maximum

107 effect of core formation on the $\mathrm{Si}$ isotopic composition of the mantle, and propose a $\mathrm{Si}$ 108 isotopic composition for the bulk Earth including different scenarios of core formation.

\section{Samples and Methods}

\subsection{Samples}

Itqyi is a coarse-grained enstatite achondrite mostly composed of enstatite (75\%)

113 and kamacite (25\%), plus traces of sulfides (Patzer et al., 2001). We obtained several

114 grams of the samples from Luc Labenne, the discoverer of Itqyi in the Western Sahara

115 Desert in 2000. This meteorite has an igneous texture with metal percolation features

116 between enstatite grains, triple junctions between enstatite grains and homogeneous

117 composition of enstatite and metal grains suggesting partial melting and

118 re-equilibration of the meteorite (Patzer et al., 2001). In order to estimate the elemental

119 distribution in the metal and silicate grains we have cut and polished a $\sim 1 \mathrm{~cm}$ section of

120 the sample (see Figure 1), mapped the sample with a field emission gun scanning

121 electron microscope (FEG-SEM) and determined chemical profiles and phase 
compositions with an electron probe microanalyzer (EPMA). We then selected and mechanically separated 6 silicate grains and 4 metal grains for Si isotopic analysis.

A sample of BHVO-2 (Hawaiian basalt, USGS standard) was analyzed following the same analytical methods for its $\mathrm{Si}$ isotopic composition, to evaluate the quality of the $\mathrm{Si}$ isotopic measurements.

\subsection{EPMA}

Metal and silicate phases of the samples were analyzed using a Cameca SX-five electron microprobe. Operating conditions were $15 \mathrm{kV}$ accelerating voltage, $10 \mathrm{nA}$ beam current and counting times of 10-20 seconds on peak and background for major and minor elements in silicate ( $\mathrm{Si}, \mathrm{Mg}, \mathrm{Fe}, \mathrm{Al}, \mathrm{Ca}, \mathrm{Na}$, Ti and $\mathrm{K})$. For trace elements ( $\mathrm{P}$, $\mathrm{Co}, \mathrm{Ni}, \mathrm{Cr}, \mathrm{V})$ in both metal and silicate, analyses were collected with $15 \mathrm{kV}$ accelerating voltage, $100 \mathrm{nA}$ beam current and counting times up to 50-100 seconds on peak and background. Diopside glass $(\mathrm{Si})$, wollastonite $(\mathrm{Ca})$, orthoclase $(\mathrm{K})$, anorthite (Al), albite (Na), rutile (Ti) and pure oxides $\left(\mathrm{Fe}_{2} \mathrm{O}_{3}, \mathrm{MgO}, \mathrm{SiO}_{2}, \mathrm{CaO}\right.$ and $\left.\mathrm{Al}_{2} \mathrm{O}_{3}\right)$ were used as standards for the major and minor elements. Pure metals were used as standards for the metal and trace elements except apatite for $\mathrm{P}$ and pyrite for S. To avoid secondary fluorescence contamination, silicate analyses were taken few tens of microns away from the metallic phases. Electron probe analyses of both metal and silicate phases are reported in Table 1.

\subsection{Isotopic measurements}

A thick section of Itqyi meteorite was crushed roughly using an agate mortar, and the enstatite and metal fragments were handpicked under a microscope. The selected 
145 flux, at $720{ }^{\circ} \mathrm{C}$ in $\mathrm{Ag}$ crucibles (99.99\% pure $\mathrm{Ag}$ at a trace metals basis). The fusion

146 cakes were placed in $30 \mathrm{~mL}$ Savillex beakers filled with 5-10 mL MQ-e $\mathrm{H}_{2} \mathrm{O}$ (18.2 $\mathrm{M} \Omega$

$147 \mathrm{~cm}^{-1}$ ), and the beakers were then placed in an ultrasonic bath for 15 minutes. The 148 samples were then transferred with pipette into pre-cleaned $50 \mathrm{~mL}$ tubes by rinsing the 149 sample-bearing $\mathrm{Ag}$ crucibles three to four times with MQ-e $\mathrm{H}_{2} \mathrm{O}$. To avoid 150 over-acidification of the solutions, only $0.10-0.30 \mathrm{~mL} 16 \mathrm{M} \mathrm{HNO}_{3}$ acid was added to 151 acidify the sample solutions till the sample solutions became clear so we reached a $\mathrm{pH}$ 152 of $\sim 1$. The metal fragments were dissolved in $8 \mathrm{M} \mathrm{HNO}_{3}$ acid, and $\mathrm{NaOH}$ was added to 153 neutralize the solutions. Over these dissolution procedures, the $\mathrm{NO}_{3}{ }^{-} / \mathrm{Si}$ ratios in the 154 dissolved solutions were monitored, based on an existing observation that the $\mathrm{Si}$ 155 contents in enstatite and metal fractions of the Itqyi meteorite were $28 \mathrm{wt} . \%$ and $3 \mathrm{wt} . \%$ 156 respectively. Solution aliquots containing $20 \mu \mathrm{g}$ Si from the Itqyi meteorite, BHVO-2 157 and NIST RM 8456 (historically, and from hereon called NBS28) were processed 158 through pre-cleaned BioRad Poly-Prep chromatography columns containing $1.8 \mathrm{~mL}$ 159 AG50X-12 (200-400 mesh) cation exchange resin to purify Si from matrix elements 160 (Georg et al., 2006). At low to neutral $\mathrm{pH}, \mathrm{Si}$ is present as either neutral or anionic 161 species. Therefore, $\mathrm{Si}$ is eluted by $\mathrm{MQ}-\mathrm{e} \mathrm{H}_{2} \mathrm{O}$ rinsing with other anions like $\mathrm{NO}_{3}{ }^{-}$, while 162 cations were kept on the columns by the AG50X-12 resin (Fujii et al., 2015). The full 163 blank for the procedure is $20 \mathrm{ng}$.

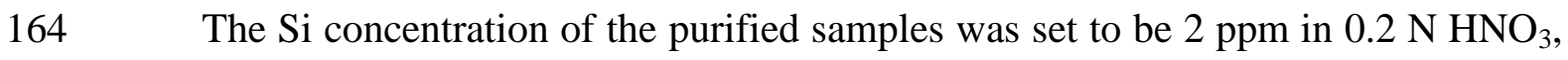
165 and were measured for Si isotopic composition on a Neptune plus multi-collector 
166 inductively-coupled-plasma mass spectrometer (MC-ICP-MS) at the Institut de

167 Physique du Globe de Paris (IPGP). Samples were analysed under "wet plasma"

168 conditions via a quartz-made spray chamber. Isobaric interferences (in particular from

$169{ }^{14} \mathrm{~N}^{16} \mathrm{O}^{+}$on ${ }^{30} \mathrm{Si}^{+}$) were avoided by measuring on the low mass peak shoulder under

170 medium mass resolution mode $(\mathrm{M} / \Delta \mathrm{M} \approx 5000)$. Under medium resolution mode, the

171 typical sensitivity for a $2 \mathrm{ppm}$ Si solution with an uptake rate of $100 \mu \mathrm{L} / \mathrm{min}$ is $10-12 \mathrm{~V}$

172 on ${ }^{28} \mathrm{Si}^{+}$, and the blank over the whole analytical session is $5-10 \mathrm{mV}$ on ${ }^{28} \mathrm{Si}^{+}$. A

173 sample-standard bracketing protocol was used in this study, i.e. measuring NBS28

174 standard once after each analysis on unknown samples. Each analysis contains 25

175 cycles with an integration time of $4 \mathrm{~s}$ for each cycle, and each sample was measured

176 four or more times. The Si isotopic results of the studied samples are reported in per mil

177 (\%o) delta notation using both ${ }^{30} \mathrm{Si} /{ }^{28} \mathrm{Si}$ and ${ }^{29} \mathrm{Si} /{ }^{28} \mathrm{Si}$ ratios of the samples relative to

178 those of the NBS28 quartz standard, i.e. $\delta^{30} \mathrm{Si}$ and $\delta^{29} \mathrm{Si}$, respectively (Table 2).

179 While the used AG50X-12 columns cannot remove $\mathrm{NO}_{3}{ }^{-}$from $\mathrm{Si}$, it has been

180 observed in this study that a mismatch of $\mathrm{NO}_{3}{ }^{-}$between sample and bracketing NBS-28

181 standard can lead to an artificial shift of sub per mil level on the measured Si isotopic

182 ratio. For instance, a purified NBS28 aliquot with $0.44 \mathrm{M} \mathrm{NO}_{3}{ }^{-}$provides a $\Delta^{30} \mathrm{Si}$ value

183 of $-0.46 \pm 0.03(2 \mathrm{SE}, \mathrm{n}=4)$ relative to another purified NBS28 aliquot with $0.2 \mathrm{M}$

$184 \mathrm{NO}_{3}^{-}$(Table 2). As such, aliquots of the fusion solution of NBS28 were doped properly

185 with $\mathrm{HNO}_{3}$ before being loaded on the AG50X-12 columns to reach similar $\mathrm{NO}_{3}{ }^{-} / \mathrm{Si}$

186 ratios as the processed aliquots of enstatite and metal samples. In detail, this match of

$187 \mathrm{NO}_{3}{ }^{-}(0.44 \mathrm{M})$ between BHVO-2 and NBS-28 successfully returned to a $\delta^{30} \mathrm{Si}$ value of 
188

$-0.25 \pm 0.05(2 \mathrm{SE}, \mathrm{n}=4)$, which is identical to the recommended value $(-0.28 \pm 0.03$; Savage et al., 2014). Measurements on the NBS28 aliquots with $0.6 \mathrm{mg}$ and $2.0 \mathrm{mg}$ dissolution masses provides $\delta^{30} \mathrm{Si}$ value of $+0.02 \pm 0.03(2 \mathrm{SE}, \mathrm{n}=4)$ and $+0.03 \pm 0.03$ (2SE, $\mathrm{n}=4$ ), respectively, implying that the $\mathrm{Si}$ isotope protocol in this study is applicable to the samples of small masses.

\section{Results}

The SEM (Figure 1) and EPMA analyzes of Si and Fe content (Table 1) confirm the igneous coarse texture and the absence or very limited elemental zoning of the meteorite (Patzer et al. 2001). The 120 degrees angles between grains are characteristic of partial melting and the percolation of metal between the enstatite grains (Figure 1) confirms the partial melting of the meteorite parent body (Patzer et al., 2001).

The EPMA Fe and Si abundance profile (Figure S1), show very homogeneous distributions within the grains. The exception being at the grain boundary, within $50-100 \mu \mathrm{m}$ (Figure S1) which may reflect contamination of the signal by secondary X-ray fluorescence from the adjacent metal or silicate grain. The rest of the grains are fairly homogeneous and we use the average composition of the EMPA profile as the average composition of the grain. For example, the metal grain \#1 (M1) is in contact with enstatite grain \#1 (E1) and enstatite grain \#2 (E2). When the first $100 \mu \mathrm{m}$ of the silicate grain is excluded we obtain an average composition for Si $(27.73 \pm 0.16$ weight $\%, 27.88 \pm 0.24$ weight $\%)$ and $\mathrm{Fe}(0.07 \pm 0.02$ weight $\%, 0.05 \pm 0.03$ weight $\%)$ content in E1 and E2 similar within 1SD. 
211 are reported in the Table 2. All the data are reported using the $\delta^{\mathrm{x}}$ Si notation, which is the

212 per mil deviation of the ${ }^{\mathrm{x}} \mathrm{Si} /{ }^{28} \mathrm{Si}$ ration from the NIST NBS28 standard $(\mathrm{x}=29$ or 30$)$.

213 All the data reported here are mass-dependent, and only $\delta^{30} \mathrm{Si}$ will discussed below.

214 When not specified, the error discussed below for the $\delta^{30} \mathrm{Si}$ are the 2 standard deviation.

The $\delta^{30} \mathrm{Si}$ of two independently processed BHVO-2 $(-0.26 \pm 0.02$ and $-0.25 \pm 0.05)$

216 are in excellent agreement with previous literature data (e.g. Abraham et al., 2008;

217 Fitoussi et al., 2009; Pringle et al., 2013; Savage et al., 2010) and the $\delta^{30} \mathrm{Si}$ of metal and

218 silicate of Itqyi is similar to what had been previously measured for other aubrite-like 219 rocks (Ziegler et al. 2010).

The $\delta^{30} \mathrm{Si}$ of the 8 silicate grains are very homogeneous with an average of $-0.57 \pm$

$2210.05(2 \mathrm{SE}, \mathrm{n}=8)$. The $\delta^{30} \mathrm{Si}$ of the 5 metal grains are slightly more variable with an 222 average of $-3.44 \pm 0.19(2 \mathrm{SE}, \mathrm{n}=5)$. When taken together and the error is propagated 223 the difference between the $\delta^{30} \mathrm{Si}$ of the silicate and metal phase $\Delta^{30} \mathrm{Si}_{\text {silicate-metal }}=2.87 \pm$ $2240.20(2 \mathrm{SE})$.

\section{Discussion}

We first estimate the $\mathrm{fO}_{2}$ and $\mathrm{T}$ of equilibrium for the metal and silicate phases. The $\mathrm{fO}_{2}$ can be determined by basing it on the oxidation equation of $\mathrm{Fe}$ :

$$
\mathrm{Fe}+\frac{1}{2} \mathrm{O}_{2}=\mathrm{FeO}, \text { Eq. } 1
$$

229 and defining the fO2 relative to its deviation from the Iron-Wustite buffer $(\Delta \mathrm{IW})$, 230 defined as:

$231 \Delta I W=2 \log \left(\frac{a_{F e O}^{\text {silicate }}}{a_{F e}^{\text {metal }}}\right)=2 \log \left(\frac{x_{F e O}^{\text {silicate }}}{x_{F e}^{\text {metal }}}\right)+2 \log \left(\frac{\gamma_{F e O}^{\text {silicate }}}{\gamma_{F e}^{\text {metal }}}\right)$. Eq. 2 
232 In equation 2, $a_{\mathrm{FeO}}^{\text {silicate }}$ and $\mathrm{a}_{\mathrm{Fe}}^{\text {metal }}$ are the activities of $\mathrm{FeO}$ in the silicate and $\mathrm{Fe}$ in

233 the metal respectively, $x_{F e O}^{\text {silicate }}$ and $x_{F e}^{\text {metal }}$ are the molar fractions of $\mathrm{FeO}$ in the

234 silicate and $\mathrm{Fe}$ in the metal respectively, and $\gamma_{\mathrm{FeO}}^{\text {silicate }}$ and $\gamma_{\mathrm{Fe}}^{\mathrm{metal}}$ are the activity

235 coefficients of $\mathrm{FeO}$ in the silicate and $\mathrm{Fe}$ in the metal. If we assume ideal behavior, the

236 activity coefficients are equal to unity, and the equation becomes:

$237 \Delta I W=2 \log \left(\frac{x_{F e O}^{\text {silicate }}}{x_{F e}^{\text {metal }}}\right) . \quad$ Eq. 3

238 By using the average composition of the silicate and metals determined by EPMA

239 (Figure $\mathrm{S} 1$ ) and by propagating the errors we obtain $\Delta \mathrm{IW}=-6.6 \pm 0.8$. This value is

240 consistent with equilibrium under very reduced conditions, similar to those estimated

241 for enstatite chondrites (Berthet et al., 2009).

242 The temperature of equilibrium can be estimated by comparing the distribution

243 coefficients of $\mathrm{Si}$ and $\mathrm{Cr}$ to experimental results. The distribution of $\mathrm{Si}$ and $\mathrm{Cr}$ between

244 metal and silicate is controlled by the following redox exchange reactions with Fe:

$$
\begin{gathered}
\left.2 \mathrm{Fe}^{\text {metal }}+\mathrm{SiO}_{2}^{\text {silicate }}=2 \mathrm{FeO} \mathrm{O}^{\text {silicate }}+\mathrm{Si}^{\text {metal }} \mathrm{Eq} .4\right) \\
\mathrm{Fe}^{\text {metal }}+\mathrm{CrO}^{\text {silicate }}=\mathrm{FeO} \text { silicate }+\mathrm{Cr}^{\text {metal }}(\text { Eq. 5). }
\end{gathered}
$$

247 The equilibrium constant of these reactions can be expanded in terms of mole fractions,

248 activity coefficients and thermodynamic constants following:

$249 \log K(S i)=\log \left(\frac{x_{S i}^{\text {metal }} / x_{\text {Silo } 2}^{\text {silicate }}}{\left(x_{F e}^{\text {metal }} / x_{\text {FeO }}^{\text {silicate }}\right)^{2}}\right)+\log \left(\frac{\gamma_{S i}^{\text {metal }} / \gamma_{\text {Silo } 2}^{\text {silicate }}}{\left(\gamma_{F e}^{\text {metal }} / \gamma_{F e O}^{\text {silicate }}\right)^{2}}\right)=a+\frac{b}{T}+c \cdot \frac{P}{T}, \quad$ Eq. 6 
$251 \log K(\mathrm{Cr})=\log \left(\frac{x_{C r}^{\text {metal }} / x_{\text {Cro }}^{\text {silicate }}}{\left(x_{F e}^{\text {metal }} / x_{\text {FeO }}^{\text {silicate }}\right)}\right)+\log \left(\frac{\gamma_{C r}^{\text {metal }} / \gamma_{C r O}^{\text {silicate }}}{\left(\gamma_{F e}^{\text {metal }} / \gamma_{\text {FeO }}^{\text {silicate }}\right)}\right)=a+\frac{b}{T}+c \cdot \frac{P}{T} \quad$ Eq. 7

253 where a, b, c are regression constants standing for the entropy, enthalpy, and volume 254 change of reactions (4) and (5) divided by the gas constant (i.e. $\Delta \mathrm{rS} \mathrm{O}^{\circ} / \mathrm{R}, \Delta \mathrm{rH}^{\circ} / \mathrm{R}$, and $255 \Delta \mathrm{rV}^{\circ} / \mathrm{R}$ respectively), $\mathrm{T}$ the temperature in $\mathrm{K}$ and $\mathrm{P}$ the pressure in GPa. The metal 256 silicate partitioning of $\mathrm{Si}$ and $\mathrm{Cr}$ have been extensively studied in high pressure and 257 high temperature experiments (e.g. Corgne et al., 2008; Fischer et al., 2015; Siebert et 258 al., 2013; Siebert et al., 2011; Wade and Wood, 2005)) leading to different expressions 259 for the parameterization of the equilibrium constants of Eq. 6 and Eq. 7. The effect of 260 pressure on the partitioning of these two elements is negligible and these datasets can 261 be suitable for modeling the conditions of equilibration of Itqyi. Using the regression 262 constants and activity models for $\mathrm{Si}$ and $\mathrm{Fe}$ in the metal from Corgne et al. (2008), 263 Siebert et al. (2013) and Fischer et al. (2015), we calculate that the measured 264 concentrations of $\mathrm{Si}$ and $\mathrm{Fe}$ in the metal and silicate phases of Itqyi equilibrated at an 265 approximate temperature of $1530 \mathrm{~K} \pm 110 \mathrm{~K}$.

266 Note here that each of the above studies treat differently the ratios of activity 267 coefficients of oxides in the silicate $\left(\gamma_{\mathrm{FeO}}^{\text {silicate }^{2}} /_{\gamma_{\mathrm{FeO}}^{\text {silicate }}}\right)$ (which represents the 268 effect of silicate composition on the partitioning of $\mathrm{Si}$ ). This parameter was either 269 considered constant as in Siebert et al. (2013) and Fischer et al. (2015) or was modeled 
270 by using the non-bridging oxygen over tetrahedra structural parameter as a proxy as in

271 Corgne et al. (2008). In an effort to best constrain the temperature of equilibrium, we

272 consider that the particular experiments conducted in Corgne et al. (2008) represent a

273 good approximation of the conditions of $\mathrm{P}-\mathrm{T}-\mathrm{fO}_{2}$, and both metal and silicate

274 compositions present during the equilibration of the Itqyi achondrite. Using regression

275 parameters from Corgne et al. (2008) only lead to the highest $\mathrm{T}$ estimate for Itqyi

276 metal-silicate equilibration of $1650 \mathrm{~K} \pm 90 \mathrm{~K}$.

277 The same above approach is followed using the $\mathrm{Cr}$ and Fe concentrations in metal 278 and silicate of Itqyi from Eq. (7). The regression constants and activity models of Eq. (7)

279 for $\mathrm{Cr}$ in metal are taken after Siebert et al. (2013) which represents an exhaustive 280 compilation of the existing dataset for Cr partitioning. Using $\mathrm{Cr}$, a $\mathrm{T}$ of $1400 \pm 240 \mathrm{~K}$ is 281 derived for the metal-silicate equilibration of Itqyi in good agreement with the $\mathrm{T}$ 282 estimated using Si partitioning data. In the following we consider the estimate based on 283 Si partitioning data rather than $\mathrm{Cr}$ as it is more precise.

284 Here we show that enstatite and metal equilibrated in Itqyi under $\mathrm{fO}_{2}$ of $\Delta \mathrm{IW}-6.6$ 285 and at a temperature of $1530 \mathrm{~K} \pm 110 \mathrm{~K}$. Note that using the approach developed in 286 Wasson (1994) leads to a similar T of equilibration of about $1500 \mathrm{~K}$. This temperature is $287 \sim 300-400$ K higher than estimated for Mount Egerton and Norton County by Ziegler et 288 al. (2010) and by combining both studies we can add a second data point with which to 289 better constrain the variation of $\Delta^{30} \mathrm{Si}_{\text {silicate-metal }}$ as a function of temperature.

290 Combining the temperature estimated above $(T=1530 \pm 110 \mathrm{~K})$ with $291 \Delta \Delta^{30} \mathrm{Si}_{\text {silicate-metal }}=2.98 \pm 0.18(2 \mathrm{SD})$ we can calculate the temperature dependence for 
292 isotopic fractionation by simply fitting a $1 / \mathrm{T}^{2}$ curve through our data point (we report 293 the error on the fit as 1SD error to be consistent with previous work Ziegler et al., 2010, 294 see Figure 2). The error includes uncertainties on the isotopic fractionation factor and 295 on the temperature of equilibrium. This returns a parametrization of:

$\Delta^{30} S i_{\text {silicate-metal }}=\frac{7.6( \pm 0.7) \times 10^{6}(1 S D)}{T^{2}}$. achondrites (Ziegler et al., 2010) $\left.\left[7.64( \pm 0.47) \times 10^{6}\right) / \mathrm{T}^{2}\right]$ and with the experiments of

300 Shahar et al. (2009) [7.45 $\left.\left.( \pm 0.41) \times 10^{6}\right) / \mathrm{T}^{2}\right]$. It is, however, of slightly larger magnitude 301 than the relationship estimated based on the experiments of Hin et al. (2014) [4.42 ( \pm 302 $\left.\left.0.05) \times 10^{6}\right) / \mathrm{T}^{2}\right]$ (Figure 2).

\section{Implications for Earth's core formation}

In many previous studies, the $\Delta^{30} \mathrm{Si}_{\text {metal-silicate }}$ parametrization has been coupled with estimates for the Si isotopic offset between bulk silicate Earth (BSE) and

307 chondrites $\left(\Delta^{30} \mathrm{Si}_{\text {BSE-chond }}\right)$ to calculate a mass-balance estimate of the amount of $\mathrm{Si}$

308 present in Earth's core (e.g. Armytage et al., 2011; Georg et al., 2007; Savage et al., 309 2014; Shahar et al., 2009). Unfortunately, these models are extremely sensitive to the 310 measured $\Delta^{30} \mathrm{Si}_{\mathrm{BSE}-\text {-chond }}$ and can predict a wide range of values, with large uncertainties, 311 which easily overlap with the outer core mass deficit based on other methods. Instead, 312 here we calculate the maximum effect of core formation on the Si isotopic composition 
313 of the BSE using our new parametrization, and based on that, estimate the Si isotope 314 composition of the bulk Earth.

315 Both single stage core formation and continuous core formation using the most 316 modern high-pressure high temperature metal/silicate partitioning data suggest that the 317 core must contain between 2 wt. \% and 9 wt. \% of Si (e.g. Fischer et al., 2015; Rubie et 318 al., 2015; Siebert et al., 2013). The permitted range for the temperature of core-mantle 319 equilibrium at the base of a magma ocean is roughly $3200-4300 \mathrm{~K}$ in order to explain 320 moderately siderophile abundances on the Earth's mantle (Badro et al., 2015; Siebert et 321 al., 2013). Using these parameters, we have calculated the maximum isotope effect that 322 core formation can have imparted on the Si isotope composition of the BSE. Following 323 the model delineated in Savage et al. (2014), for a single-stage core formation model, 324 the effect of core formation on the $\mathrm{Si}$ isotopic composition is plotted in Figure 3 as a 325 function of the increase in $\mathrm{Si}$ in the core (from 2 to $9 \mathrm{wt} \%$ ) (parameters used in the 326 model are indicated in the figure caption). The two lines plotted in Figure 3 are the 327 calculated $\delta^{30} \mathrm{Si}$ isotope composition of the bulk Earth, for two temperatures of 328 equilibration $(3200 \mathrm{~K}$ and $4300 \mathrm{~K})$. If the core contains less than $7 \% \mathrm{Si}$, as suggested by 329 several studies based on elemental partitioning and physical properties (Antonangeli et 330 al., 2010; Badro et al., 2015), the bulk Earth must be heavier than any chondrites.

331 As can be seen even for the most extreme calculated case (3200 K equilibration 332 temperature and $9 \% \mathrm{Si}$ in the core) this cannot account for $0.14 \%$ of the difference 333 between the chondrites and the Earth. In other words, the Si composition of the bulk 334 Earth may be at the lightest $-0.43 \%$ (note this value is extreme as core formation is 
unlikely to have remained at $3200 \mathrm{mK}$ throughout Earth's differentiation). If we use less

extreme parameters (e.g. T $=3800 \mathrm{~K}, \mathrm{Si}$ in core $=6 \mathrm{wt} . \%$ ) we calculate a bulk Earth value of $\sim-0.35 \%$. This value $\sim 0.10 \%$ heavier than ordinary and carbonaceous chondrites and $\sim 0.20 \%$ heavier than enstatite chondrites (see Figure 4) (Armytage et al., 2011; Fitoussi et al., 2009; Savage and Moynier, 2013; Zambardi et al., 2013).

The difference between the Si isotopic composition of the bulk silicate Earth and

341 chondrites may be due to either 1) inaccuracies in the estimation of the $\mathrm{Si}$ isotopic 342 composition of the BSE or 2) that the bulk Earth has a non-chondritic Si isotopic 343 composition.

\section{Do we have an accurate estimate of the Si isotope composition of Earth's mantle?}

345 Silicon isotopes are very weakly fractionated by partial melting and fractional 346 crystallization. For example Savage et al. (2010) showed that ultramafic various mantle 347 rocks, MORBs and Island Arc basalts have all similar isotopic composition within error 348 and estimated the BSE to be at $-0.29 \pm 0.08 \%$. These findings are very consistent with 349 most other studies (e.g. Armytage et al., 2011; Fitoussi et al., 2009; Zambardi et al., 350 2013; Ziegler et al. 2010) and combining all these data together Savage et al. (2014) 351 recommend a $\delta^{30} \mathrm{Si}=-0.29 \pm 0.07 \%$ for the composition of the BSE, which seems to be 352 a robust estimate of the Earth's upper mantle composition. More recently, it has been 353 shown that high degree partial melting from the Archean mantle, komatiites, have 354 identical composition (-0.29 $\pm 0.02 \%$, Deng et al., 2019), giving further support to 355 previous estimates of the BSE. 
It should be kept in mind, however, that all the above BSE estimates are based on samples derived from the upper mantle, and it has been posited that the lower mantle may have a different isotopic composition. For example, it was proposed by Huang et al. (2014), based on theoretical calculations, that lower mantle high pressure phases

360 (bridgmanite) should be isotopically lighter than upper mantle phases (olivines, 361 pyroxenes). Based on these calculations, Huang et al. (2014) suggested that the lower 362 mantle could be 0.1 permil lighter than the upper mantle. Considering the proportion of 363 lower ( 75\%) and upper ( 25\%) mantle, Huang et al. (2014) calculated that it could 364 be possible for the BSE to have a $\delta^{30} \mathrm{Si}$ of $\sim-0.37$. It is however uncertain whether such 365 isotopic heterogeneity could fully be preserved in the lower mantle on the scale of 4.6 366 Ga or homogenized by global scale convection and still be present today (e.g. 367 Brandenburg et al., 2008; Stixrude and Lithgow-Bertelloni, 2012). In further evidence 368 against an isotopically layered mantle, Pringle et al. (2016) reported a large set of data 369 for Ocean Island basalts from various localities, which were mostly undistinguishable $370(-0.32+/-0.09)$ from the isotopic composition reported by Savage et al. (2010). While 371 some samples measured by Pringle et al. (2016) were found to have $\delta^{30} \mathrm{Si}$ values $0.1 \%$ 372 lower than upper mantle samples, these were not common, and although this paper 373 argued for the existence of some mantle heterogeneity (introduced by recycling of 374 crust), given the paucity of these isotopically "light" samples, it is unlikely that the bulk 375 of the lower mantle will be drastically different from the upper mantle, at least not to the 376 level of difference needed to bring the BSE to chondritic values (Pringle et al., 2016). 

accurate and representative of the BSE, and therefore explore the possibility that the bulk Earth is non-chondritic in terms of its $\mathrm{Si}$ isotope composition.

\section{The Bulk Earth has a non-chondritic Si isotope composition}

We here consider that the bulk Earth has a heavier Si isotope composition than all known chondrites groups. First, there is always the possibility that none of the chondrites from our collection represent the $\mathrm{Si}$ isotope composition of the Earth's precursors materials - and that all of the Earth-chondrites accreted to the Earth (Drake and Righter, 2002), and/or that Earth is an end-member Si isotopic composition, similar to the arguments made for r-process anomalies (Fischer-Godde and Kleine, 2017). However, given the small degree of variation in the Si isotope composition of all 389 chondrite groups, it more likely that Si that accreted to form the Earth started in the 390 solar nebula with a "chondritic" composition, and was subsequently fractionated prior 391 to or during accretion. Pringle et al. (2014) found that angrite meteorites, that are most 392 likely sampling a small parent body than Earth, are isotopically heavier than chondrites 393 by $\sim 0.1 \%$. Since these samples are among the most volatile depleted samples in the 394 solar system, it was suggested that the parent body of the angrite meteorites 395 experienced volatile loss that resulted in a preferential loss of the lighter Si isotopes. 396 Pringle et al. (2014) suggested that this volatile loss could also have affected Earth's 397 precursors materials and would therefore increase the bulk Earth $\mathrm{Si}$ isotopic 398 composition. It was further suggested that based on model of volatile loss from 
399 planetesimals and isotopic fractionation under equilibrium that $\sim 15 \%$ Si loss could 400 explain the composition of the Earth within the first Myrs of the Solar System (Young 401 et al., 2019). Alternatively, Dauphas et al. (2015) invoke Si isotopes fractionation 402 between condensing solids and the nebula gas, and that Earth's precursors were 403 depleted in refractory phases (forsterite), decreasing its $\mathrm{Mg} / \mathrm{Si}$ ratio and increasing $\delta^{30} \mathrm{Si}$. 404 This alternative scenario may also explain part of the Si isotopic fractionation of the 405 bulk Earth, however, it should be noted that there is a weak (coefficient of 406 determination, $\mathrm{R}^{2}=0.5$ ) relationship between the $\delta^{30} \mathrm{Si}$ and the $\mathrm{Mg} / \mathrm{Si}$ ratio for primitive 407 meteorites and the Earth (see Figure 4).

408 We therefore suggest that Earth's precursor material underwent volatile depletion 409 during its accretion such that its $\mathrm{Si}$ isotope composition was fractionated from the 410 "chondritic" baseline, to a heavier isotopic composition (Hin et al., 2017; Pringle et al., 411 2014; Young et al., 2019) . Core formation then further drove this bulk Earth value to its 412 current composition, which we measure today.

\section{Conclusions}

415 We have used an enstatite meteorite, Itqyi, as a natural experiment to determine the

416 Si isotopic fractionation factor between metal and silicate. To reach this goal, we have 417 performed a petrological and isotopic study of the meteorite in order to determine the 418 temperature of equilibrium as well as the Si isotopic composition between the phases. 419 We find that the dependence of Si isotopes with temperature to be $1530 \mathrm{~K} \pm 110 \mathrm{~K}$. We 420 use this dependence together with various core formation model and composition to 
421 estimate the bulk Earth $\delta^{30} \mathrm{Si}$. We show that the bulk Earth must be isotopically heavier 422 than any chondrites groups, save that the lower mantle would be isotopically light due 423 to the preservation of bridgmanite/melt isotopic fractionation in the magma ocean. In 424 agreement with previous study we suggest that volatility have shaped the composition 425 of the Earth's precursors and modified the final composition of the bulk Earth 426 compared to its original building blocks.

428 Acknowledgments: We deeply thank the two reviewers for insightful comments and 429 for correcting important mistakes and Raj Dasgupta for efficient editing. This paper has 430 been written during a visit of FM to CUG Wuhan in October 2019. FM thanks 431 Yongsheng Liu and Jiubin Chen for making this visit possible. F. M. acknowledges 432 funding from the European Research Council under the H2020 framework 433 program/ERC grant agreement (\#637503-Pristine). 


\section{References}

436 Abraham, K., Opfergelt, S., Fripiat, F., Cavagna, A., deJong, J.T.M., Foley, S.F., 437 André, L., Cardinal, D., 2008. $\delta^{30} \mathrm{Si}$ and $\delta^{29} \mathrm{Si}$ determinations on USGS BHVO-1and 438 BHVO-2 reference materials with a newc onfiguration on a $\mathrm{Nu}$ plasma multi-collector

Antonangeli, D., Siebert, J., Badro, J., Farber, D., Fiquet, G., Morard, G., Ryerson, .F., 2010. Composition of the Earth's inner core from high-pressure sound velocity measurements in Fe-Ni-Si alloys. Earth Planet. Sci. Lett. 295, 292-296.

Armytage, R.M.G., Georg, R., Savage, P., Williams, H., Halliday, A., 2011. Silicon isotopes in meteorites and planetary core formation. Geochim. Cosmochim. Acta 75, 3662-3676.

Badro, J., Brodholt, J., Piet, H., Siebert, J., Ryerson, F.J., 2015. Core formation and core composition from coupled geochemical and geophysical constraints. Proc. Natl. Acad Sci U S A 112, 12310-12314.

Berthet, S., Malavergne, V., Righter, K., 2009. Melting of the Indarch meteorite (EH4 chondrite) at $1 \mathrm{GPa}$ and variable oxygen fugacity: Implications for early planetary differentiation processes. Geochim Cosmochim Acta 73, 6402-6420.

Birch, F., 1964. Density and composition of mantle and core. J.Geophys.Res. 69, 4377-4388.

Bonnand, P., Halliday, A., 2018. Oxidized conditions in iron meteorite parent bodies. Nature Geoscience 11, 401-404.

Brandenburg, J., Hauri, E., van Keken, P., Ballentine, C., 2008. A multiple system study of the geochemical evolution of the mantle with force-balanced plates and thermochemical effects. Earth Planet. Sci. Lett. 276, 1-13.

Corgne, A., Keshav, S., Wood, B.J., McDonough, W.F., Fei, Y., 2008. Metal-silicate partitioning and constraints on core composition and oxygen fugacity during Earth accretion. Geochim. Cosmochim. Acta 72, 574.

Creech, J., Baker, J., Handler, M., Lorand, J., Storey, M., Moynier, F., Bizzarro, M., 2017. Late accretion history of terrestrial planets inferred from stable isotopes. Geochemical Perspective Letters 2, 94-104.

Dauphas, N., Poitrasson, F., Burkhardt, C., Kobayashi, H., Kurozawa, K., 2015. Planetary and meteoritic $\mathrm{Mg} / \mathrm{Si}$ and $\delta^{30} \mathrm{Si}$ variations inherited from solar nebula chemistry. Earth Planet. Sci. Lett. 427, 236-248. 
Deng, Z., Chaussidon, M., Guitreau, M., Puchtel, I., Dauphas, N., Moynier, F., 2019. An oceanic subduction origin for Archaean granitoids revealed by silicon isotopes. Nature Geoscience 12, 774-778.

Drake, M.J., Righter, K., 2002. Determining the composition of the Earth. Nature 416, 39-44.

Fischer, R., Nakajima, Y., Campbell, A.J., Frost, D.J., Harries, D., Langenhorst, F., Muajima, N., Polock, K., Rubie, D.C., 2015. High pressure metal-silicate partitioning of Ni, Co, V, Cr, Si, and O. Geochim. Cosmochim. Acta 167, 177-194.

Fischer-Godde, M., Kleine, T., 2017. Ruthenium isotopic evidence for an inner Solar System origin of the late veneer. Nature 541, 525-527.

Fitoussi, C., Bourdon, B., 2012. Silicon Isotope Evidence Against an Enstatite Chondrite Earth. Science 335, 1477-1480.

Fitoussi, C., Bourdon, B., Kleine, T., Oberli, F., Reynolds, B.C., 2009. Si isotope systematics of meteorites and terrestrial peridotites: implications for $\mathrm{Mg} / \mathrm{Si}$ fractionation in the solar nebula and for Si in the Earth's core. Earth Planet. Sci. Lett. 287, 77-85.

Fujii, T., Pringle, E., Chaussidon, M., Moynier, F., 2015. Isotope fractionation of Si in protonation/deprotonation reaction of silicic acid: A new $\mathrm{pH}$ proxy. Geochim. Cosmochim. Acta 168, 193-205.

Georg, B., Reynolds, B.C., Frank, M., Halliday, A., 2006. New sample preparation techniques for the determination of $\mathrm{Si}$ isotopic compositions using MC-ICPMS. Chem. Geol. 235, 95-104.

Georg, R.B., Halliday, A.N., Schauble, E.A., Reynolds, B.C., 2007. Silicon in the Earth's core. Nature 447, 1102-1106.

Hin, R., Burkhardt, C., Schmidt, M., Bourdon, B., Kleine, T., 2013. Experimental evidence for Mo isotope fractionation between metal and silicate liquids. Earth Planet. Sci. Lett. 379, 38-48.

Hin, R., Fitoussi, C., Schmidt, M., Bourdon, B., 2014. Experimental determination of the $\mathrm{Si}$ isotope fractionation factor between liquid metal and liquid silicate. Earth Planet. Sci. Lett. 387, 55-66.

Hin, R.C., Coath, C.D., Carter, P.J., Nimmo, F., Lai, Y.J., Pogge von Strandmann, P.A.E., Willbold, M., Leinhardt, Z.M., Walter, M.J., Elliott, T., 2017. Magnesium 
isotope evidence that accretional vapour loss shapes planetary compositions. Nature $549,511-515$.

Hopp, T., Fischer-Godde, M., Kleine, T., 2018. Ruthenium isotope fractionation in protoplanetary cores. Geochim. Cosmochim. Acta 223, 75-89.

Huang, F., Wu, Z., Huang, S., Wu, F.Y., 2014. First-principles calculations of equilibrium silicon isotope fractionation among mantle minerals. Geochim. Cosmochim. Acta 140, 509-520.

Mahan, B., Siebert, J., Blanchard, I., Badro, J., Kubik, E., Sossi, P., Moynier, F., 2018. Investigating Earth's Formation History Through Copper and Sulfur Metal- Silicate Partitioning During Core- Mantle Differentiation. J. Geophys. Res.123, 8349-8363

Patzer, A., Hill, D.H., Boynton, W.V., 2001. Itqiy: A metal-rich enstatite meteorite with achondritic texture. Meteorit. Planet. Sci. 36, 1495-1505.

Patzer, A., Hill, D.H., Boynton, W.V., Frank, L., Schultz, L., Hull, T., McHargue, L., Franchi, I.A., 2002. Itqiy: A study of noble gases and oxygen isotopes including its terrestrial age and a comparison with Zaklodzie. Meteorit. Planet. Sci. 37, 823-833.

Poitrasson, F. 2017, Silicon isotope geochemistry. In review in mineralogy and geochemistry vol. 82, Non traditional stable isotopes. Editors: Teng, Dauphas, Watkins, publisher MSA, pp.289-344

Pringle, E., Moynier, F., Savage, P., JAckson, M., Moreira, M., Day, J., 2016. Silicon isotopes reveal recycled altered oceanic crust in the mantle sources of Ocean Island Basalts. Geochim. Cosmochim. Acta 189, 282-295.

Pringle, E., Savage, P., Badro, J., Barrat, J.A., Moynier, F., 2013. Redox state during core formation on asteroid 4-Vesta. Earth Planet. Sci. Lett. 373, 75-82.

Pringle, E.A., Moynier, F., Savage, P.S., Badro, J., Barrat, J.A., 2014. Silicon isotopes in angrites and volatile loss in planetesimals. Proc Natl Acad Sci U S A 111, 17029-17032.

Righter, K., Drake, M.J., 2003. Partition Coefficients at High Pressure and Temperature, Treatise on Geochemistry, Volume 2. Editor: Richard W. Carlson. Executive Editors: Heinrich D. Holland and Karl K. Turekian. pp. 568. ISBN 0-08-043751-6. Elsevier, 2003., p.425-449, pp. 425-449.

Roskosz, M., Luais, B., Watson, H.C., Toplis, M.J., Alexander, C.M.O.D., Mysen, B.O., 2006. Experimental quantification of the fractionation of Fe isotopes during metal segregation from a silicate melt. Earth Planet. Sci. Lett. 248, 851-867. 
567 Rubie, D.C., Jacobson, S.A., Morbidelli, A., O’Brien, D.P., Young, E.D., de Vries, J., 568 Nimmo, F., Palme, H., Frost, D.J., 2015. Accretion and differentiation of the terrestrial 569 planets with implications for the compositions of early-formed Solar System bodies and accretion of water. Icarus 248, 89-108.

Savage, P., Armytage, R., Georg, B., Halliday, A., 2014. High temperature silicon isotope geochemistry. Lithos 190, 500-519.

Savage, P., Georg, B., Armytage, R., Williams, H., Halliday, A., 2010. Silicon isotope homogeneity in the mantle. Earth Planet. Sci. Lett. 15, 139-146.

Savage, P., Moynier, F., 2013. Silicon isotopic variation in enstatite meteorites: Clues to their origin and Earth-forming material. Earth Planet. Sci. Lett. 361, 487-496.

Savage, P., Moynier, F., Chen, H., Shofner, G., Siebert, J., Badro, J., Puchtel, I.S., 2015. Copper isotope evidence for large-scale sulphide fractionation during Earth's differentiation. Geochemical Perspective Letters 1, 53-64.

Shahar, A., Elardo, S., Macris, C., 2017. Equilibrium fractionation of non-traditional stable isotopes: an experimental perspective, In review in mineralogy and geochemistry vol. 82, Non traditional stable isotopes. Editors: Teng, Dauphas, Watkins, publisher MSA, pp. 65-83.

Shahar, A., Ziegler, K., Young, E.D., Ricolleau, A., SChauble, E.A., Fei, Y.W., 2009. Experimentally determined Si isotope fractionation between silicate and Fe metal and implications for Earth's core formation. Earth Planet. Sci. Lett. 288, 228-234.

Siebert, J., Badro, J., Antonangeli, D., Ryerson, R., 2013. Terrestrial accretion under oxidizing conditions. Science 338, 1194-1197.

Siebert, J., Corgne, A., Ryerson, F.J., 2011. Systematics of metal-silicate partitioning for many siderophile elements applied to Earth's core formation. Geochim.

601 Sikdar, J., Rai, V., 2020. Si-Mg isotopes in enstatite chondrites and accretion of reduced planetary bodies. Scientific Reports, 1273.

Stixrude, L., Lithgow-Bertelloni, C., 2012. Geophysics of chemical heterogeneity in the mantle. Ann. Rev. Earth Planet. Sci. 40, 569-595. 
610 Young, E.D., Shahar, A., Schlichting, H., Schauble, E., Tang, H., Labidi, J., 2019.

611 Near-equilibrium isotope fractionation during planetesimal evaporation. Icarus 323,

612 1-15.

613

614 Zambardi, T., Poitrasson, F., Corgne, A., Meheut, M., Quitté, G., Anand, M., 2013.

615 Silicon isotope variations in the inner solar system: Implications for planetary

616 formation, differentiation and composition. Geochim Cosmochim Acta 121, 67-83.

617

618 Ziegler, K., Young, E.D., Schauble, E.A., Wasson, J.T., 2010. Metal-silicate silicon 619 isotope fractionation in enstatite meteorites and constraints on Earth's core formation. 620 Earth Planet. Sci. Lett. 295, 487-496.

621

622

623 
Figure Captions:

625

626

627

628

629

630

631

632

633

634

635

636

637

638

639

640

641

642

643

644

Figure 1: Backscattered image of the Itqyi sample utilized in this study. The white phases represents the Fe-rich metal while the dark phases represents the enstatite minerals.

Figure 2: $\Delta \Delta^{30} \mathrm{Si}_{\text {silicate-metal }}$ as a function of temperature. The $\Delta^{30} \mathrm{Si}_{\text {silicate-metal }}$ obtained for Itqyi is reported. The two curves represent the estimated relation of $\Delta^{30} \mathrm{Si}_{\text {silicate-metal }}$ vs temperature from Shahar et al. (2009) and Hin et al. (2014).

Figure 3: Modeled silicon isotopic composition of the bulk Earth as a function of $\mathrm{Si}$ content of the core for two temperatures of equilibrium (3200K and 4300K) using the isotopic fractionation factor determined in this study. For the parameters used in the model we used: Mass fraction of the $\mathrm{BSE}=0.67$, mass fraction of the core $=0.33$, mass fraction of $\mathrm{Si}$ in the $\mathrm{BSE}=0.212, \delta^{30} \mathrm{Si}(\mathrm{BSE})=-0.29 \pm 0.08$ (Savage et al. 2014), $\Delta^{30} i_{\text {silicate-metal }}=\frac{7.6( \pm 0.7) \times 10^{6}}{T^{2}}$.

Figure 4: $\delta^{30} \mathrm{Si}$ as a function of $\mathrm{Mg} / \mathrm{Si}$ ratio for various chondrites and the Earth (using the updated $\delta^{30} \mathrm{Si}$ for the bulk Earth calculated in the present study). The Si isotopic composition of the meteorites are from literature (Armytage et al., 2011; Fitoussi and Bourdon, 2012; Fitoussi et al., 2009; Zambardi et al., 2013).

Table Captions:

Table 1: Electron probe microanalyses of enstatite and metal phases.

Table 2: Silicon isotopic composition of the different samples analyzed in this study. 
645 Table 1. Electron probe microanalyses of enstatite and metal phases.

646

\begin{tabular}{cccc}
\hline $\mathrm{Wt} \%$ & Itqiy (Enstatite) & $\mathrm{Wt} \%$ & Itqiy (Metal) \\
\hline $\mathrm{MgO}$ & $37.050(1.098)$ & $\mathrm{Fe}$ & $88.960(0.660)$ \\
$\mathrm{SiO}_{2}$ & $59.404(0.383)$ & $\mathrm{Si}$ & $2.436(0.129)$ \\
$\mathrm{Al}_{2} \mathrm{O}_{3}$ & $0.560(0.022)$ & $\mathrm{Ni}$ & $4.441(0.175)$ \\
$\mathrm{FeO}$ & $0.061(0.018)$ & $\mathrm{Cr}$ & $0.210(0.015)$ \\
$\mathrm{CaO}$ & $1.616(0.052)$ & $\mathrm{Co}$ & $0.284(0.012)$ \\
$\mathrm{TiO}_{2}$ & $0.029(0.017)$ & $\mathrm{Mg}$ & $0.136(0.011)$ \\
$\mathrm{Cr}_{2} \mathrm{O}_{3}$ & $0.032(0.010)$ & & \\
\hline $\mathrm{Total}$ & $98.752(0.731)$ & $\mathrm{Total}$ & $96.467(1.770)$
\end{tabular}


649 Table 2. Silicon isotopic composition of the different samples analyzed in this study.

\begin{tabular}{|c|c|c|c|c|c|c|c|c|}
\hline Samples & $\delta^{29} \mathrm{Si}$ & 2SD & 2SE & $\delta^{30} \mathrm{Si}$ & 2SD & 2SE & $\mathbf{N}$ & Description \\
\hline NBS28 & 0.00 & 0.08 & 0.01 & 0.00 & 0.10 & 0.01 & 154 & $26.3 \mathrm{mg}$ \\
\hline NBS28-test 1 & 0.01 & 0.05 & 0.03 & 0.02 & 0.06 & 0.03 & 4 & $0.6 \mathrm{mg}, \mathrm{NO}_{3}-\mathrm{match}$ \\
\hline NBS28-test 2 & 0.04 & 0.07 & 0.03 & 0.03 & 0.06 & 0.03 & 4 & $2.0 \mathrm{mg}, \mathrm{NO}_{3}-\mathrm{match}$ \\
\hline BHVO-2 & -0.14 & 0.06 & 0.01 & -0.26 & 0.14 & 0.02 & 35 & $16.0 \mathrm{mg}, \mathrm{NO}_{3}^{-}$match \\
\hline BHVO-2 & -0.13 & 0.11 & 0.05 & -0.25 & 0.11 & 0.05 & 4 & $\mathrm{NO}_{3}^{-}$match: $0.44 \mathrm{~N}^{2}$ versus $0.44 \mathrm{~N} \mathrm{NO}_{3}$ \\
\hline Enstatite_01 & -0.30 & 0.06 & 0.03 & -0.64 & 0.16 & 0.08 & 4 & $\mathrm{NO}_{3}{ }^{-}$match \\
\hline Enstatite_02 & -0.32 & 0.05 & 0.03 & -0.69 & 0.09 & 0.05 & 4 & $\mathrm{NO}_{3}{ }^{-}$match \\
\hline Enstatite_03 & -0.27 & 0.07 & 0.04 & -0.52 & 0.07 & 0.04 & 4 & $\mathrm{NO}_{3}{ }^{-}$match \\
\hline Enstatite_04 & -0.27 & 0.04 & 0.02 & -0.50 & 0.07 & 0.04 & 4 & $\mathrm{NO}_{3}{ }^{-}$match \\
\hline Enstatite_05 & -0.25 & 0.04 & 0.02 & -0.49 & 0.07 & 0.04 & 4 & $\mathrm{NO}_{3}{ }^{-}$match \\
\hline Enstatite_06 & -0.26 & 0.03 & 0.02 & -0.58 & 0.03 & 0.01 & 4 & $\mathrm{NO}_{3}{ }^{-}$match \\
\hline Enstatite_07 & -0.27 & 0.09 & 0.05 & -0.56 & 0.08 & 0.04 & 4 & $\mathrm{NO}_{3}{ }^{-}$match \\
\hline Enstatite_08 & -0.28 & 0.04 & 0.02 & -0.56 & 0.16 & 0.08 & 4 & $\mathrm{NO}_{3}{ }^{-}$match \\
\hline Metal_01 & -1.71 & 0.06 & 0.03 & -3.56 & 0.02 & 0.01 & 4 & $\mathrm{NO}_{3}^{-}$match \\
\hline Metal_02 & -1.61 & 0.15 & 0.07 & -3.16 & 0.30 & 0.15 & 4 & $\mathrm{NO}_{3}^{-}$match \\
\hline Metal_03 & -1.71 & 0.04 & 0.02 & -3.35 & 0.10 & 0.05 & 4 & $\mathrm{NO}_{3}^{-}$match \\
\hline Metal_04 & -1.93 & 0.06 & 0.03 & -3.74 & 0.04 & 0.02 & 4 & $\mathrm{NO}_{3}{ }^{-}$match \\
\hline Metal_05 & -1.76 & 0.07 & 0.04 & -3.41 & 0.05 & 0.03 & 4 & $\mathrm{NO}_{3}{ }^{-}$match \\
\hline Average Enstatite & -0.28 & 0.04 & 0.02 & -0.57 & 0.14 & 0.05 & 8 & \\
\hline Average Metal & -1.74 & 0.23 & 0.10 & -3.44 & 0.44 & 0.19 & 5 & \\
\hline
\end{tabular}


Click here to download Figure: Figure 1.pdf

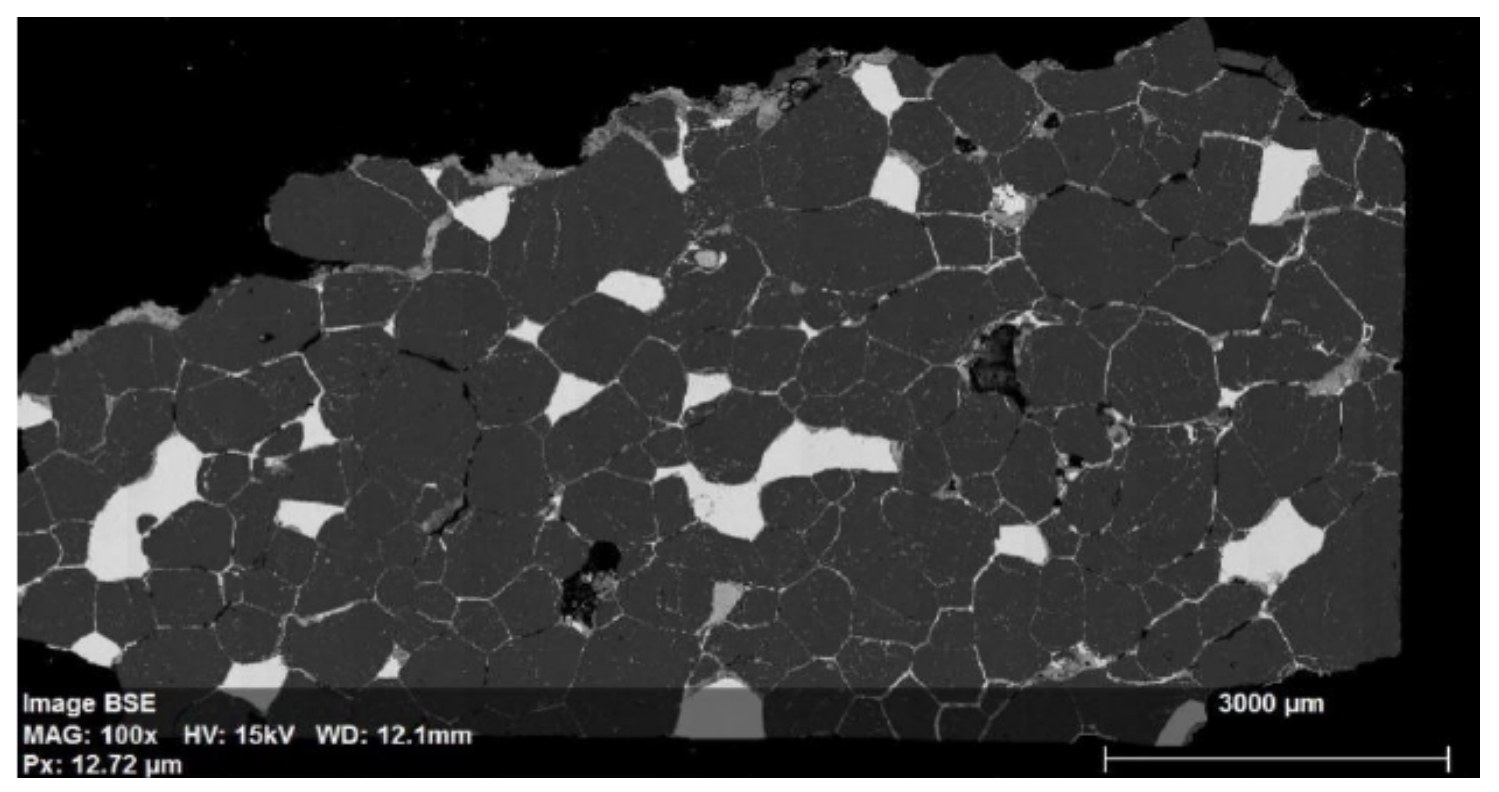


Figure 2
Click here to download Figure: Figure2.pdf

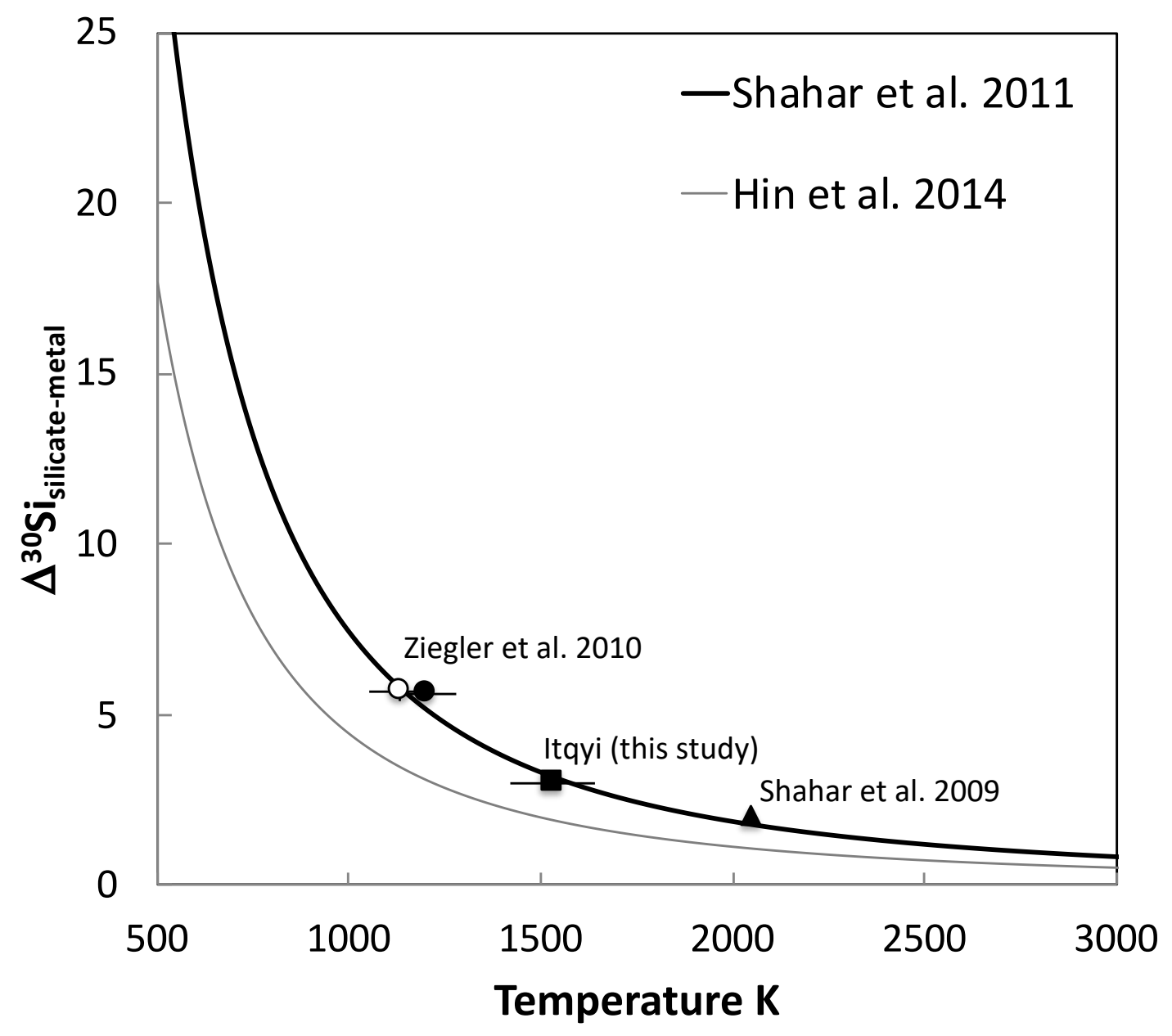




\section{Figure 3}

Click here to download Figure: Si isotope model new.pdf

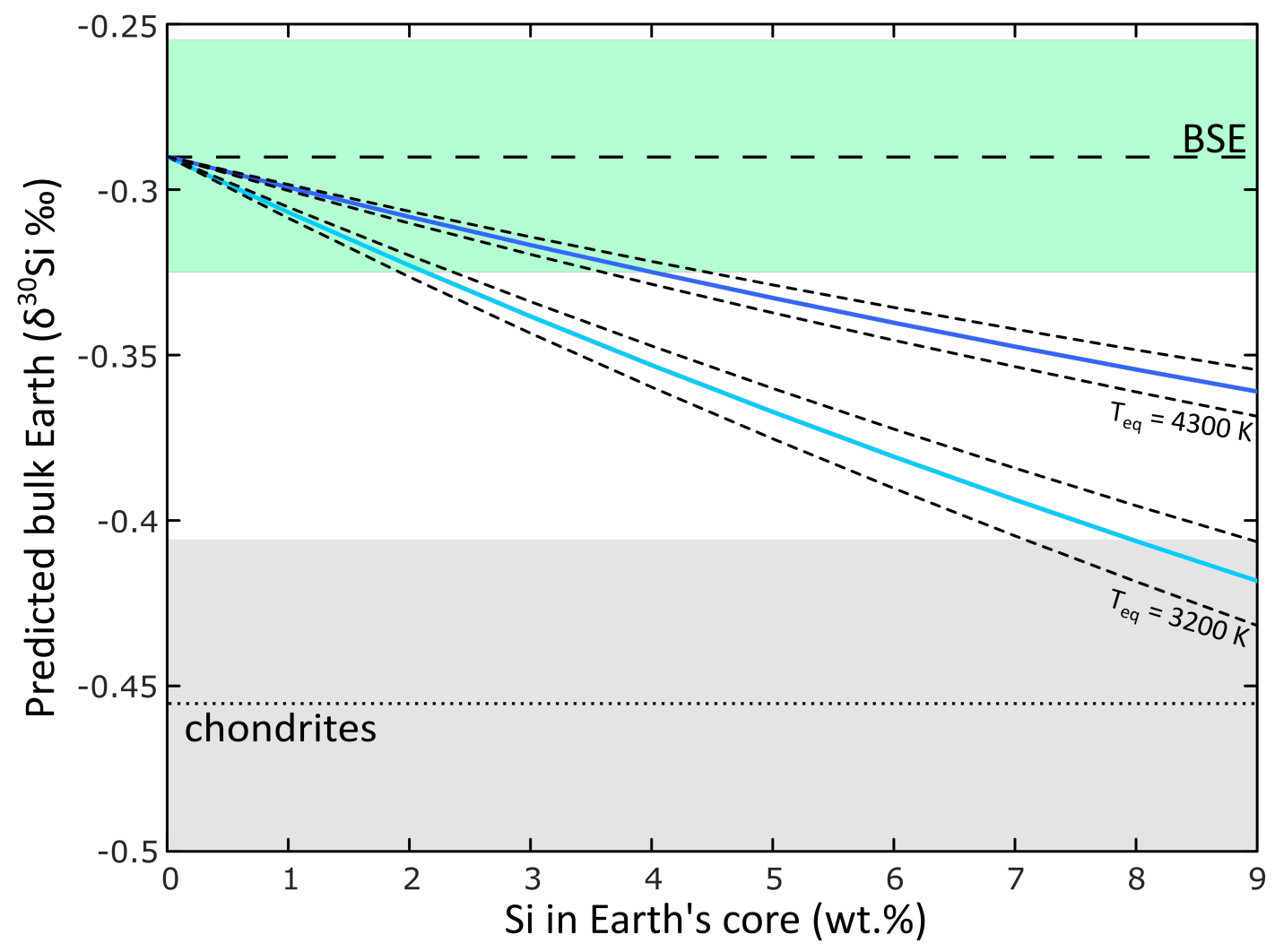




\section{Figure 4}

Click here to download Figure: Fiogure4.pdf

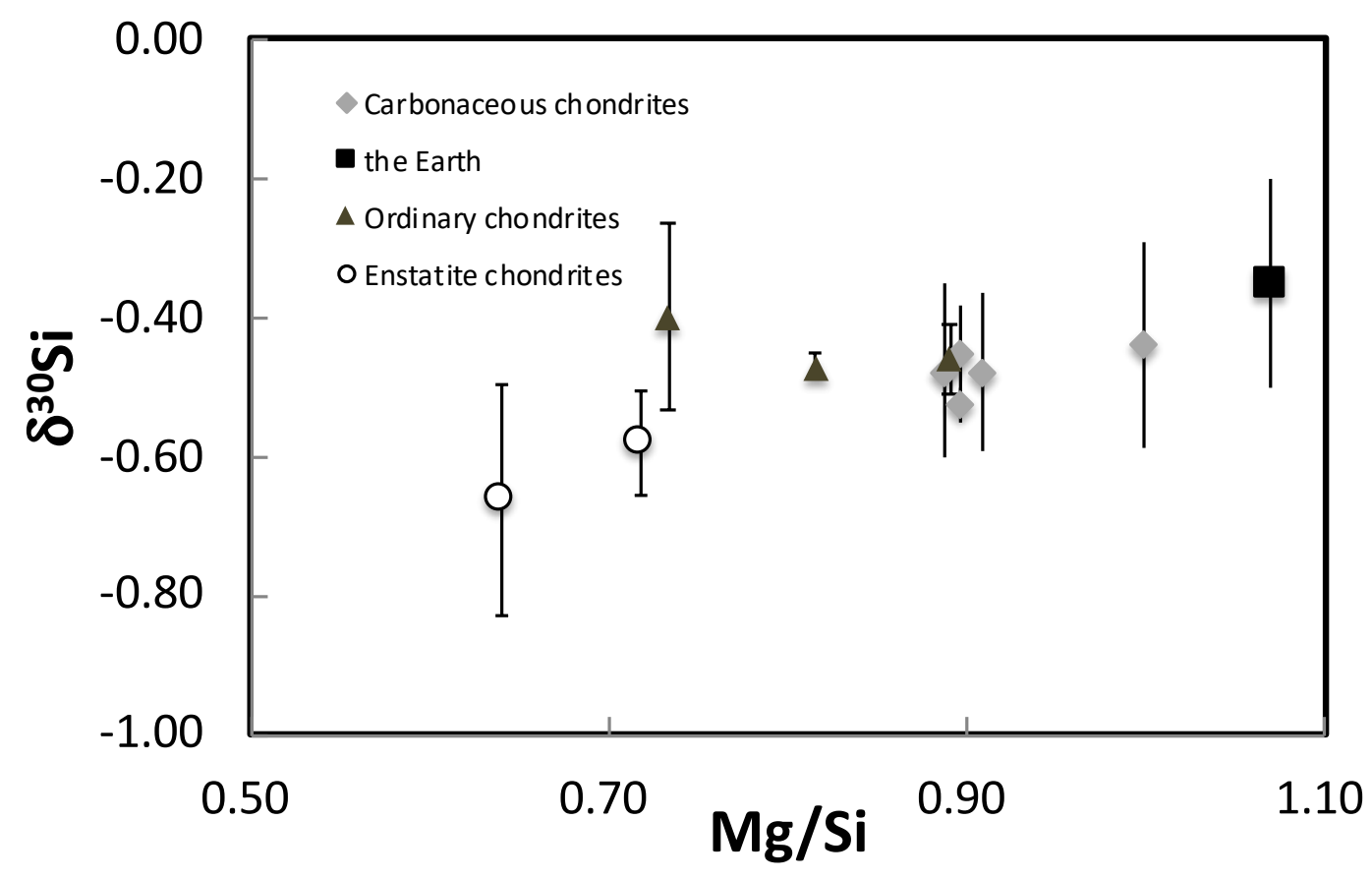



Supplementary material for online publication only
Click here to download Supplementary material for

Click here to download Supplementary material for online publication only: Supplementary Materials.docx (a) o X

(1)

(1)
. .

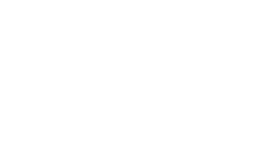

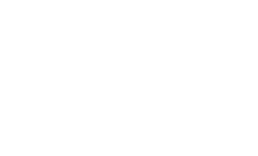
.

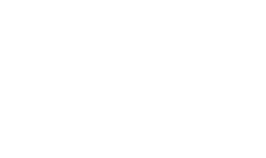
(1) (1) (1) (1)

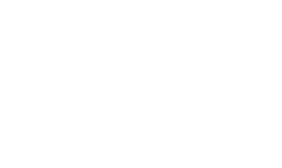




\section{Declaration of interests}

$\bigotimes$ The authors declare that they have no known competing financial interests or personal relationships that could have appeared to influence the work reported in this paper.

$\square$ The authors declare the following financial interests/personal relationships which may be considered as potential competing interests:

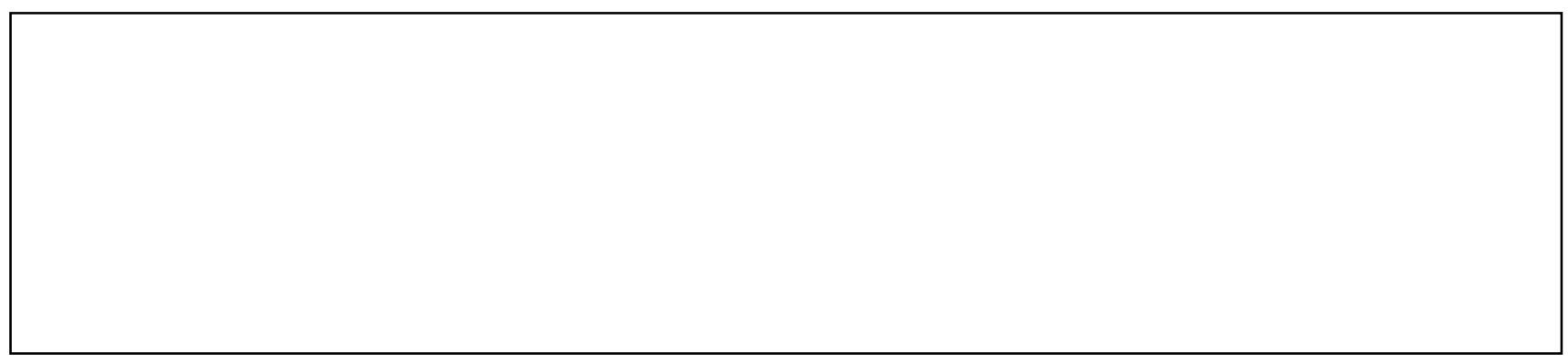


Frederic Moynier: Conceptualization, interpretation, writing. Zhengbin Deng: methodology, interpretation, writing. Ariane Lanteri: methodology. Rayssa Martins: methodology. Marc Chaussidon: interpretation, writing. Paul Savage: interpretation, writing. Julien Savage: interpretation, writing. 